\title{
Generalized Unsöld theorem and radial distribution function for hydrogenic orbitals
}

\author{
S.M. Blinder \\ Department of Chemistry, University of Michigan, Ann Arbor, \\ MI 48109-1055, USA
}

Received 11 September 1992; revised 1 February 1993

\begin{abstract}
A generalization of Unsöld's theorem is derived, giving the sum over both $l$ and $m$ for products of hydrogenic functions with given $n$. A generalized radial distribution function $D_{n}(r)$ is introduced and its properties discussed. As $n \rightarrow \infty, D_{n}(r)$ approaches a universal reduced form, for which an empirical representation is given.
\end{abstract}

By Unsöld's theorem [1] the sum over all $m$ states for a shell of hydrogenlike orbitals reduces to a spherically-symmetrical function:

$$
\sum_{m=-l}^{l}\left|\psi_{n l m}(r, \theta, \phi)\right|^{2}=\rho_{n l}(r) .
$$

For a pure Coulomb potential, the different $l$ states for a given $n$ are also degenerate. We present in this paper an explicit form for the sum over both $l$ and $m$ for hydrogenic orbitals, viz.,

$$
\sum_{l=0}^{n-1} \rho_{n l}(r)=\rho_{n}(\boldsymbol{r})
$$

The result is not entirely new $[2,3]$ but we will develop it here in greater detail. We encounter summations over $\rho_{n}(r)$ in computation of the Coulomb statistical density matrix $[4,5]$. The bound-state contribution is explicitly

$$
\sum_{n=1}^{\infty} \rho_{n}(r) \mathrm{e}^{-\beta E_{n}}
$$

We note that, in recent experiments [6], hydrogen atoms have been excited by electric fields to high Rydberg states in excess of $n=70$.

The most straightforward derivation of what we call the "generalized Unsöld 
theorem" makes use of the Coulomb Green's function. First derived in closed form by Hostler [7], the Green's function can be expressed as follows [8]:

$$
G^{+}\left(\boldsymbol{r}_{1}, \boldsymbol{r}_{2}, E\right)=G^{+}(x, y, k)=-\frac{1}{\pi(x-y)}\left(\frac{\partial}{\partial x}-\frac{\partial}{\partial y}\right) g^{+}(x, y, k),
$$

where

$$
g^{+}(x, y, k)=(\mathrm{i} k)^{-1} \Gamma(1-\mathrm{i} \nu) M_{\mathrm{i} \nu}(-\mathrm{i} k y) W_{\mathrm{i} \nu}(-\mathrm{i} k x),
$$

the latter function representing a pseudo one-dimensional Coulomb system. The coordinate variables $x$ and $y$ are defined by

$$
x \equiv r_{1}+r_{2}+r_{12}, \quad y \equiv r_{1}+r_{2}-r_{12} .
$$

The energy is related to the wavenumber $k$ by

$$
E=\hbar^{2} k^{2} / 2 m=k^{2} / 2
$$

in the atomic units: $\hbar=m=e=1$. We also introduce the parameter

$$
\nu \equiv Z / k \text {. }
$$

$M$ and $W$ are Whittaker functions as defined by Buchholz [9]. For brevity we write $M_{\mathrm{i} \nu}$ for $M_{\mathrm{i} \nu, 1 / 2}$ and $W_{\mathrm{i} \nu}$ for $W_{\mathrm{i} \nu, 1 / 2}$.

We recall the spectral representation of the Green's function, running over both discrete and continuum eigenstates:

$$
\begin{aligned}
G\left(\boldsymbol{r}_{1}, \boldsymbol{r}_{2}, E\right)= & \sum_{n, l, m} \frac{\psi_{n, l, m}\left(\boldsymbol{r}_{1}\right) \psi_{n, l, m}^{*}\left(\boldsymbol{r}_{2}\right)}{E-E_{n}} \\
& +\sum_{l, m} \int_{0}^{\infty} \frac{\psi_{k, l, m}\left(\boldsymbol{r}_{1}\right) \psi_{k, l, m}^{*}\left(\boldsymbol{r}_{2}\right)}{E-k^{2} / 2} \mathrm{~d} k
\end{aligned}
$$

with $\operatorname{Im} E>0$ for $G^{+}$. The gamma function has poles at $\nu=-\mathrm{i} n, n=1,2, \ldots$, corresponding to the discrete Coulomb spectrum $E_{n}=-Z^{2} / 2 n^{2}$. The corresponding residues of the Green's function can evidently be identified with the sum over the discrete eigenstates of a given $n$. We define the density function

$$
\rho_{n}\left(\boldsymbol{r}_{1}, \boldsymbol{r}_{2}\right)=\sum_{l, m} \psi_{n, l, m}\left(\boldsymbol{r}_{1}\right) \psi_{n, l, m}^{*}\left(\boldsymbol{r}_{2}\right)
$$

so that

$$
\rho_{n}\left(\boldsymbol{r}_{1}, \boldsymbol{r}_{2}\right)=-\left(\frac{Z_{n}}{\pi}\right)(x-y)^{-1}\left(\frac{\partial}{\partial x}-\frac{\partial}{\partial y}\right) M_{n}\left(Z_{n} x\right) M_{n}\left(Z_{n} y\right) .
$$

We have introduced the abbreviation $Z_{n} \equiv Z / n$. The Whittaker function for integer $n$ are related to Laguerre functions as follows:

$$
M_{n}(z)=\frac{z}{n} \mathrm{e}^{-z / 2} L_{n-1}^{(1)}(z)=z \mathrm{e}^{-z / 2} F_{1}(n-1 ; 2 ; z) .
$$


Also

$$
W_{n}(z)=(-)^{n-1} n ! M_{n}(z) .
$$

This result also follows from the discrete part of the Coulomb propagator [5], but the derivation given above is more illuminating.

Note that the density functions, like the Green's function, have reduced to a function of just the two configuration variables $x$ and $y$. Explicitly, the first three density functions are

$$
\begin{aligned}
& \rho_{1}=\left(Z^{3} / \pi\right) \mathrm{e}^{-Z \xi}, \\
& \rho_{2}=\left(Z^{3} / 8 \pi\right)\left(1-\frac{1}{2} Z \xi+\frac{1}{8} Z^{2} \eta^{2}\right) \mathrm{e}^{-Z \xi / 2}, \\
& \rho_{3}=\frac{Z^{3}}{27 \pi}\left[1-\frac{2}{3} Z \xi+\frac{2}{27} Z^{2}\left(\xi^{2}+2 \eta^{2}\right)-\frac{2}{81} Z^{3} \xi \eta^{2}+\frac{1}{972} Z^{4} \eta^{4}\right] \mathrm{e}^{-Z \xi / 3},
\end{aligned}
$$

where

$$
\xi \equiv(x+y) / 2, \quad \eta \equiv \sqrt{x y} .
$$

Application of the above formulas to hybrid atomic orbitals and open-shell computations has been suggested [8].

The analog of (11) for continuum states works out to

$$
\begin{aligned}
\rho_{k}\left(\boldsymbol{r}_{1}, \boldsymbol{r}_{2}\right) & =\sum_{l, m} \psi_{k, l, m}\left(\boldsymbol{r}_{1}\right) \psi_{k, l, m}^{*}\left(\boldsymbol{r}_{2}\right) \\
& =\frac{1}{2 \pi^{2}} \mathrm{e}^{\pi \nu}|\Gamma(1-\mathrm{i} \nu)|^{2}(x-y)^{-1}\left(\frac{\partial}{\partial x}-\frac{\partial}{\partial y}\right) M_{\mathrm{i} \nu}(-\mathrm{i} k x) M_{\mathrm{i} \nu}(-\mathrm{i} k y) .
\end{aligned}
$$

We now specialize $\rho_{n}\left(\boldsymbol{r}_{1}, \boldsymbol{r}_{2}\right)$ to the limit $\boldsymbol{r}_{1}=\boldsymbol{r}_{2}=\boldsymbol{r}$ or $x=y=2 r$. The result is

$$
\rho_{n}(r)=\frac{Z_{n}^{3}}{\pi}\left[M_{n}^{\prime}\left(2 Z_{n} r\right)^{2}-M_{n}\left(2 Z_{n} r\right) M_{n}^{\prime \prime}\left(2 Z_{n} r\right)\right] .
$$

We can define the generalized radial distribution functions

$$
D_{n}(r) \equiv 4 \pi r^{2} \rho_{n}(r) \text {, }
$$

which are normalized according to

$$
\int_{0}^{\infty} D_{n}(r) \mathrm{d} r=n^{2}
$$

reflecting the orbital degeneracy of the corresponding energy level $E_{n}$.

In fig. 1 we show the radial distribution functions for $n=1,2,5,10,20$ and 50, with $Z=1$. As $n$ increases, the function evidently approaches a universal reduced 

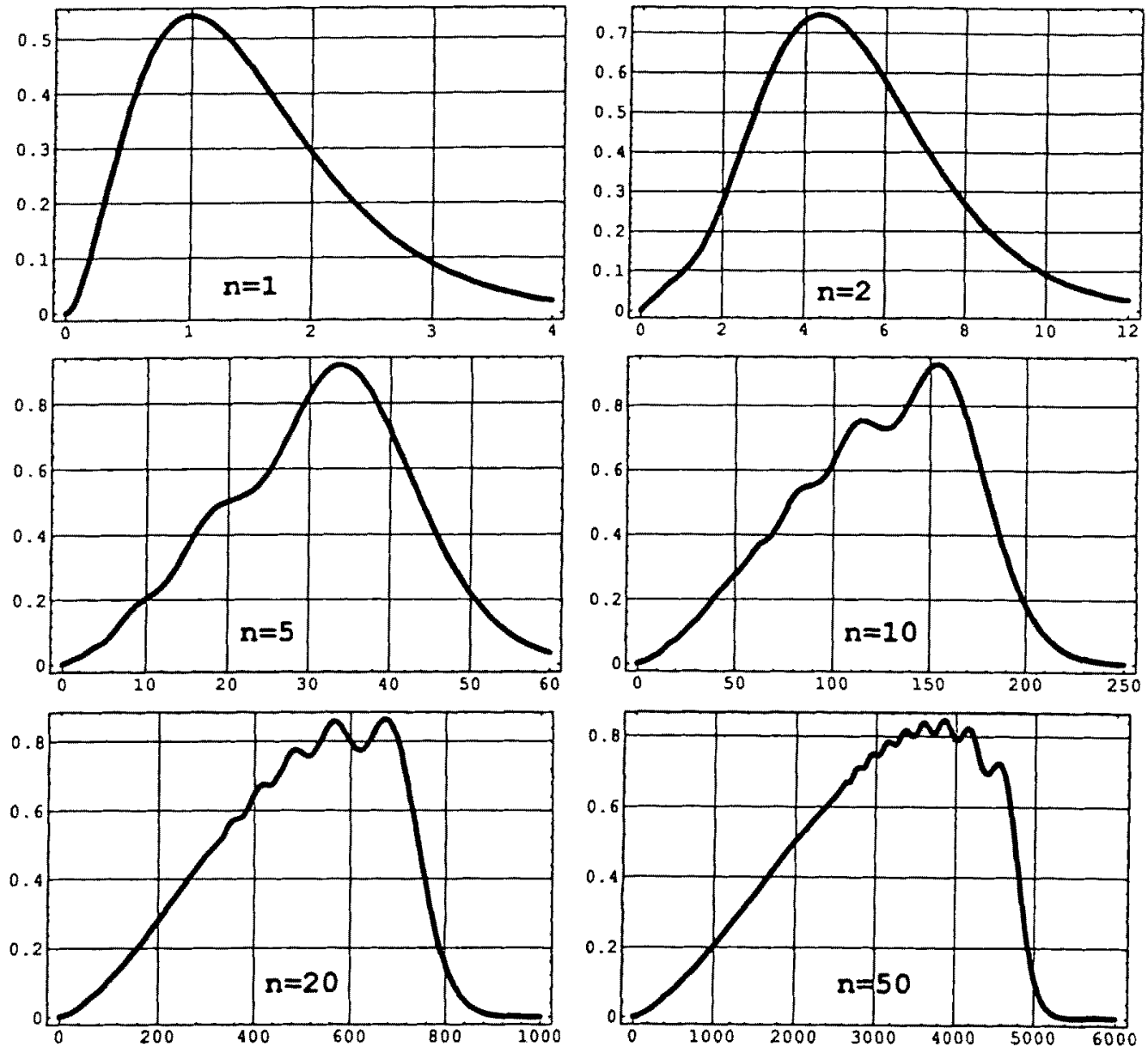

Fig. 1. Generalized radial distributions functions $D_{n}(r)$ for $Z=1, n=1,2,5,10,20,50$. Radius $r$ in bohrs.

form, apart from some small oscillations. $D_{n}(r)$ exhibits a maximum value $\approx 0.835$ near $r \approx 1.5 n^{2} / Z$. Remarkably, this maximum value is never exceeded for any value of $n$. The radial distribution functions decay rapidly beyond $r=2 n^{2} / Z$ and drop effectively to zero for $r \approx 2.1 n^{2} / Z$. We were not able to derive an analytic representation for the $n \rightarrow \infty$ asymptotic form of $D_{n}(r)$. However, an approximate representation is suggested by the asymptotic behavior of the Whittaker function for $n \gg Z r \gg 1$, viz., 


$$
M_{n}(2 Z r / n) \sim \frac{1}{n} \sqrt{2 Z r} J_{1}(\sqrt{8 Z r}) \sim \frac{1}{\sqrt{\pi} n}(2 Z r)^{1 / 4} \cos (\sqrt{8 Z r}-3 \pi / 4),
$$

which leads to

$$
D_{n}(r) \sim \frac{Z}{\pi}\left(\frac{2 Z r}{n^{2}}\right)^{3 / 2}
$$

This suggests the empirical representation:

$$
D_{n}(r) \approx \frac{Z}{\pi} \rho^{3 / 2}\left(1+a \rho+b \rho^{2}+c \rho^{3}+d \rho^{4}\right), \quad \rho \equiv 2 Z r / n^{2} .
$$

The best least-squares fit for $0 \leq r \leq 2.1 n^{2} / Z$ is obtained with $a=-0.267973$, $b=0.0863527, c=-0.0135544, d=-0.00126477$. The dashed curve in fig. 2 shows the fitted function with these parameters compared to the accurate radial distribution function for $n=100$.

\section{$\mathbf{D}_{\mathbf{n}}(\mathbf{r})$}

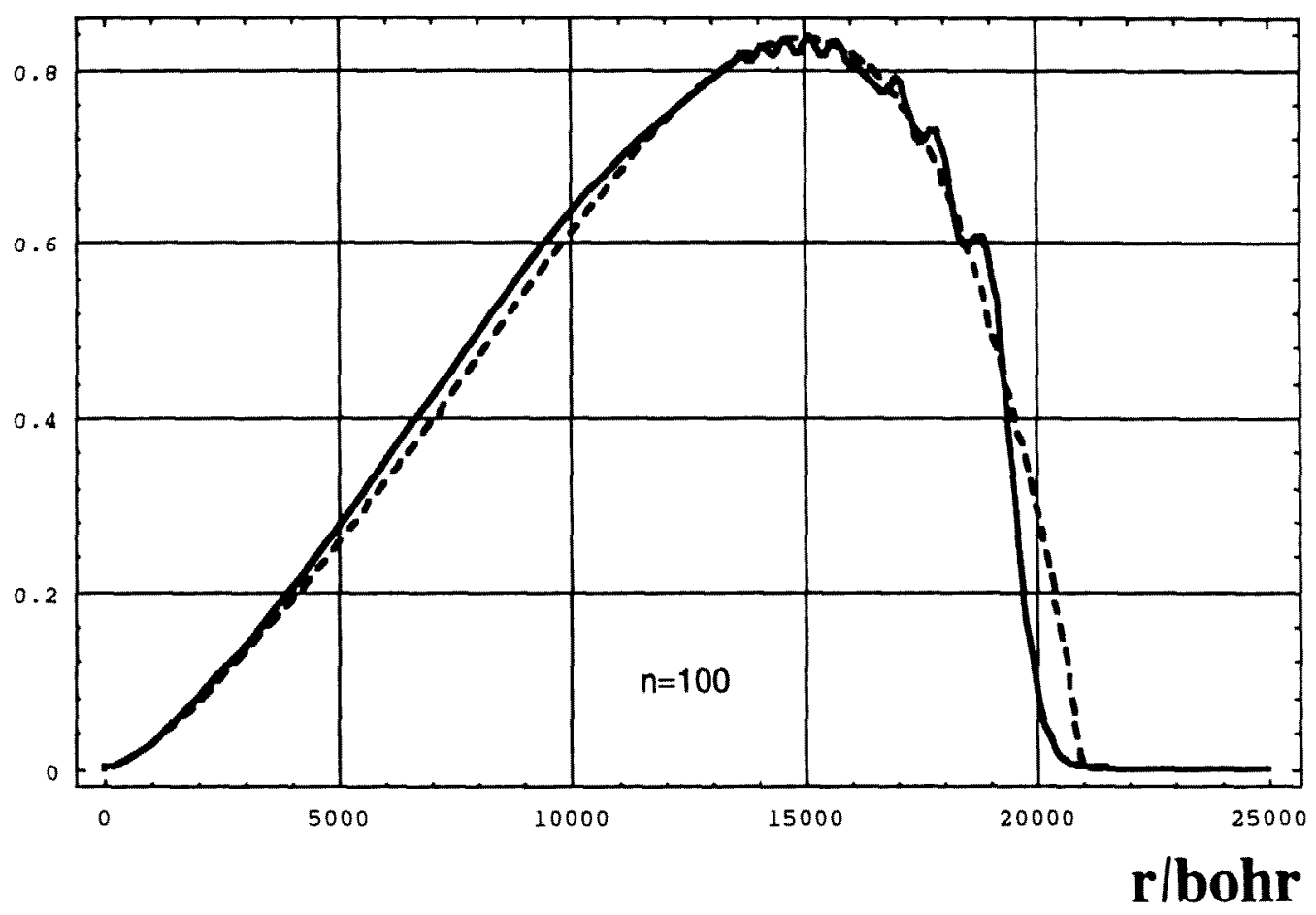

Fig. 2. Solid curve: radial distributions function for $n=100$. Dashed curve: best fit using eq. (21). 


\section{References}

[1] A. Unsöld, Ann. Phys. 82 (1927) 355.

[2] L. Hostler, J. Math. Phys. 11 (1970) 2966.

[3] S.M. Blinder, Bull. Am. Phys. Soc. 28 (1983) 1309.

[4] E.L. Pollock, Commun. Comput. Phys. 52 (1988) 49.

[5] S.M. Blinder, Phys. Rev. A43 (1991) 13.

[6] J.N. Bardsley and B. Sundaram, Phys. Rev. A32 (1985) 689.

[7] L. Hostler and R.H. Pratt, Phys. Rev. Lett. 10 (1963) 469;

L. Hostler, J. Math. Phys. 5 (1964) 591.

[8] S.M. Blinder, Int. J. Quant. Chem. S18 (1984) 293 and references therein.

[9] H. Buchholz, The Confluent Hypergeometric Function (Springer, New York, 1969). 